\title{
Étude relative à l'apprentissage collectif généré par les ateliers
}

\author{
Marc-Urbain Proulx, directeur \\ Guillaume Jouhannet, assistant de recherche \\ Centre de recherche sur le développement territorial (CRDT) \\ Université du Québec à Chicoutimi
}

\section{ATELIER «PACTE SOCIAL DE L'ALUMINIUM »}

\section{Cette première analyse représente une} synthèse des éléments dont nous disposions avant le forum, c'est pourquoi elle va servir de base pour comparer à ce que nous allons obtenir en plus à l'issue de l'atelier.

Afin de mesurer l'apprentissage collectif généré par un des ateliers du forum « pacte social de l'aluminium » nous nous appuierons sur deux analyses SWOT. La première s'appuie sur la grappe québécoise de l'aluminium. Elle n'est pas focalisée sur la Vallée de l'Aluminium du Saguenay -Lac-StJean mais comprend un nombre important d'éléments qui s'appliquent à la Vallée. Cette première analyse représente une synthèse des éléments dont nous disposions avant le forum, c'est pourquoi elle va servir de base pour comparer à ce que nous allons obtenir en plus à l'issue de l'atelier. La seconde analyse est une version adaptée des éléments recueillis durant l'atelier. Elle comprend une synthèse des points relevés dans le premier SWOT et des points nouveaux abordés durant l'atelier.

Ces deux travaux sont synthétisés dans l'analyse cidessous qui reprend les éléments dont nous disposions déjà (en rouge) et les apports de l'atelier (en vert). Certains éléments ont été fusionnés de façon à ne comptabiliser que les éléments réellement nouveaux. Ainsi il est possible de mesurer les éléments soulevés par les participants à l'atelier afin de quantifier l'apprentissage collectif qui en résulte.

\section{Analyse SWOT des éléments listés avant et après l'atelier}

\begin{tabular}{|c|c|}
\hline 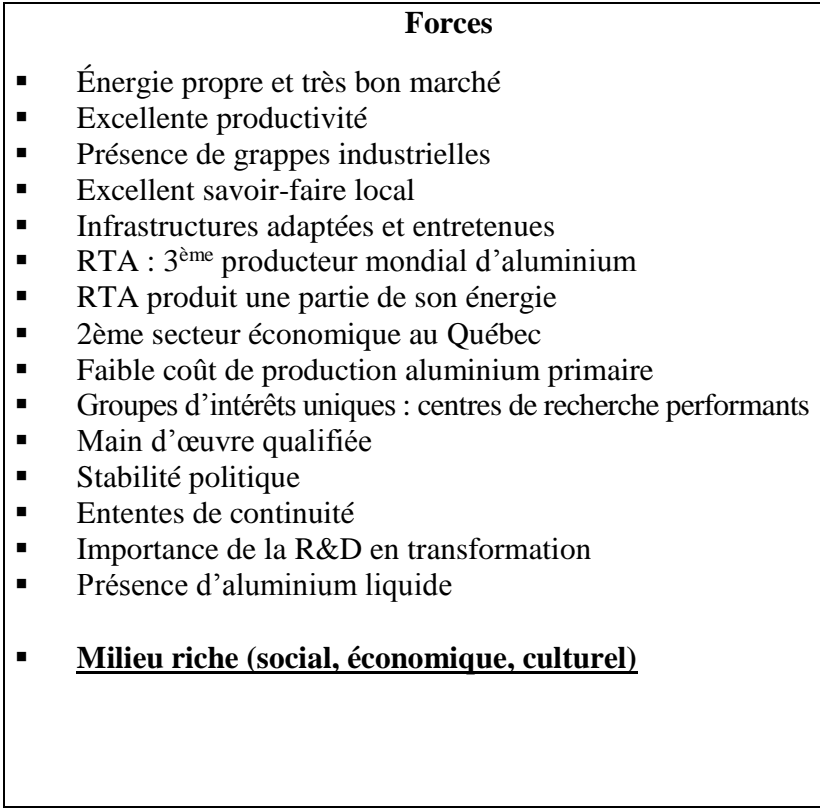 & \begin{tabular}{ll} 
& \multicolumn{1}{c}{ Faiblesses } \\
- & Coût élevé de la main d'œuvre \\
- & Installations anciennes \\
- & Coût importation bauxite guinéenne \\
- & Transformation délaissée par RTA \\
- & Coût de production alumine important \\
- & Aluminium primaire exporté sans transfo. \\
- & Prime Midwest : perte avantage concurrentiel \\
- & Inaccessibilité métal petits transformateurs \\
- & Manque de fonds (capitaux de risque) \\
- & Alu Québec renforce les grands centres \\
- & Perte de contacts avec Rio Tinto \\
- & $\frac{\text { Part de l'énergie appartenant à RTA }}{\text { - }}$ \\
- & $\frac{\text { Pous-traitants vulnérables devant RTA }}{\text { Perte de réciprocité entre RTA et la région }}$ \\
- & $\frac{\text { Manque de transparence dans les ententes }}{\text { Manque de solidarité régionale }}$ \\
- & $\frac{\text { Absence d'instance régionale }}{\text { Manque d'appui gouvernemental }}$ \\
- & $\frac{\text { Absence de réflexion collective régionale }}{\text { - }}$ \\
\end{tabular} \\
\hline
\end{tabular}




\begin{tabular}{|c|c|}
\hline 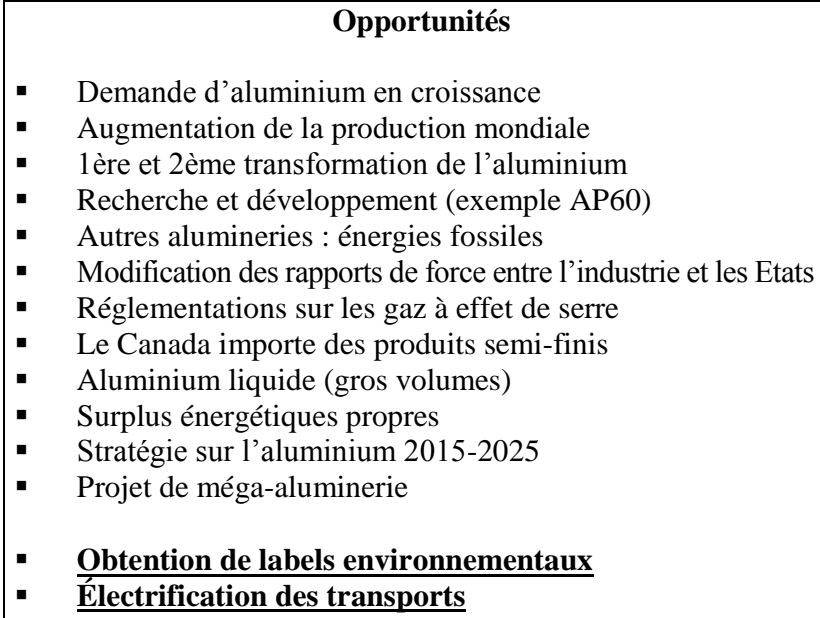 & \begin{tabular}{ll} 
& \multicolumn{1}{c}{ Menaces } \\
- & Pays émergeants (méga-alumineries) \\
- & Compétition interrégionale \\
- & Manque de politique gouvernementale claire \\
- & Le « Buy American Act $~$ \\
- & Instabilité politique fournisseurs de bauxite \\
- & Vision stratégique de Rio Tinto inconnue \\
$-\quad$ Dumping \\
$-\quad$ Division entre les acteurs \\
- $\quad$ Capacité de navigation sur le Saguenay
\end{tabular} \\
\hline
\end{tabular}

\section{1 Étude comparative}

L'étude comparative entre les deux travaux fait ressortir les éléments suivants :

Forces : 1 élément nouveau $(15 / 1=+6,7 \%)$

Faiblesses : 10 éléments nouveaux $(10 / 10=$ $+100 \%$ )

Opportunités : 2 éléments nouveaux $(2 / 12=$ $+16,7 \%$ )

Menaces : 5 éléments nouveaux $(5 / 7=+71,4 \%)$

Ce travail fait ressortir le fait que ce sont principalement sur les faiblesses et les menaces que les intervenants se sont concentrés. Ainsi si quelques points ont été ajoutés en ce qui concerne les forces $(+6,7 \%)$ et les opportunités $(+16,7 \%)$ les résultats obtenus en termes de faiblesses $(+100 \%)$ et menaces $(+71,4 \%)$ permettent de quantifier les inquiétudes qui pèsent l'avenir de la Vallée de
l'Aluminium. Certains éléments comme les problématiques de capacité de circulation nautique étant des éléments inédits qui n'avaient pas été abordés lors des travaux précédents.

On note également le caractère très local des apports de l'atelier. L'intégralité des nouveaux éléments, à l'exception des opportunités, concerne des problématiques propres à la région. Les rares éléments plus globaux évoqués pouvant être rattachés à des éléments déjà présents dans le premier SWOT.

\section{Ce travail fait ressortir le fait que ce sont principalement sur les faiblesses et les menaces que les intervenants se sont concentrés.}

\section{ATELIER « FISCALITÉ DES BARRAGES ET CENTRALES »}

Pour ce second atelier les SWOT avant et après atelier ont été dissociés de façon à ce que seuls les éléments évoqués durant l'atelier n'apparaissent dans le deuxième SWOT. L'étude est réalisée de la même façon que pour l'atelier précédent.

\section{Analyse SWOT des éléments listés avant et après l'atelier}

\begin{tabular}{|l|ll|}
\hline \multicolumn{1}{|c|}{ Forces } & \multicolumn{1}{c|}{ Faiblesses } \\
- Immense bassin du SLSJ & - Non-inscription au rôle foncier \\
- 323 centrales de production & - Fiscalité basées sur l'arbitraire du passé \\
- Majorité des des installations appartiennent aux & - Perceptions fiscales inégales, incohérentes \\
- entreprises auto-consommatrices & - $\begin{array}{l}\text { Bases du calcul des perceptions désuètes } \\
\text { - Concentration d'auto-consom. au SLSJ }\end{array}$ & - $\begin{array}{l}\text { Méconnaissance de l'arbitraire des } \\
\text { perceptions fiscales actuelles }\end{array}$ \\
\hline
\end{tabular}




\begin{tabular}{|c|c|}
\hline 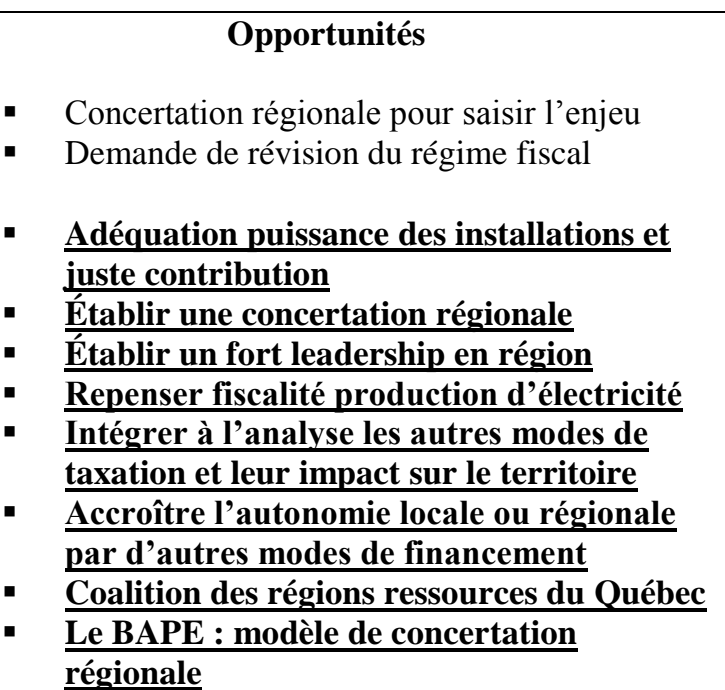 & $\begin{array}{l}\text { Menaces } \\
\text { - } \begin{array}{l}\text { Insatisfaction accrue (municipalités, } \\
\text { entreprises, citoyens) }\end{array} \\
\text { - } \frac{\text { Réforme engendre discorde }}{\text { La «peur de réveiller le chat qui dort » }} \\
\text { - } \frac{\text { Déséquilibre des forces en présence }}{}\end{array}$ \\
\hline
\end{tabular}

\section{1 Étude comparative}

\section{Dans cet atelier l'apprentissage collectif mis en lumière dans le deuxième SWOT a été très important.}

L'étude comparative entre les deux travaux fait ressortir les éléments suivants :

Forces : 2 éléments nouveaux $(2 / 4=+50 \%)$

Faiblesses : 3 éléments nouveaux $(3 / 3=$ $+100 \%$ )

Opportunités : 8 éléments nouveaux $(8 / 2=$ $+400 \%$ )

Menaces : 3 éléments nouveaux $(3 / 1=+300 \%)$
Dans cet atelier l'apprentissage collectif mis en lumière dans le deuxième SWOT a été très important. Ce sont principalement sur les opportunités que les participants se sont concentrés avec 8 éléments nouveaux représentant une augmentation de $400 \%$. Les menaces et faiblesses présentent de fortes augmentations $(+100 \%$ et $+300 \%)$ cependant elles sont principalement dues au faible nombre d'éléments présents dans le premier SWOT. Enfin les forces ont été moins évoquées $(+50 \%)$

Il est intéressant de constater que contrairement au premier atelier les réflexions, et principalement celles portant sur les opportunités incluent l'ensemble du Québec et s'éloignent de la problématique presque exclusivement régionale relevée dans le premier travail.

\section{ATELIER « NOUVEAU MARCHÉ RÉGIONAL DE LA SOUS-TRAITANCE »}

L'atelier sur le nouveau marché régional de la sous-traitance est résumé dans deux SWOT successifs, le second compilant les données présentes avant et après l'atelier.

\section{Analyse SWOT des éléments listés avant et après l'atelier}

\begin{tabular}{|l|l|}
\hline \multicolumn{1}{|c|}{ Forces } & \multicolumn{1}{c|}{ Faiblesses } \\
- Meilleurs coûts pour RT & - - 3000 emplois directs \\
- Opportunités entrepreneuriales & - Perte de masse salariale \\
- Classe ouvrière intermédiaire & - Faible profitabilité \\
- 450 à 500 emplois indirects & - Achats hors région \\
- Syndicalisation à $60 \%$ & - Perte opportunités manufacturière \\
\hline
\end{tabular}




\begin{tabular}{|l|l|}
\hline \multicolumn{1}{|c|}{ Opportunités } & \multicolumn{1}{c|}{ Menaces } \\
- Nouvelle attractivité ouvrière & - Vices du monopsone \\
- Nouvelle classe d'affaires & - Perte de qualité ouvrière \\
- Moins de couts d'implantation & - Perte de productivité \\
- Diversification économique sous-traitants & - Érosion de l'entrepreneuriat \\
- Impulsion entrepreneuriale & - Peu de contrôle économique \\
- Regroupement des intérêts & \\
\hline
\end{tabular}

\section{1 Étude comparative}

L'étude comparative entre les deux travaux fait ressortir les éléments suivants :

Opportunités : 2 éléments nouveaux $(2 / 4=+50 \%)$

Contrairement aux deux précédents ateliers, celui traitant du nouveau marché régional de la soustraitance est demeuré très limité en termes d'apprentissage collectif. Ainsi bien que les idées soient formulées différemment, les forces, faiblesses et menaces ne comportent aucun élément inédit et c'est uniquement dans les opportunités que nous pouvons relever deux éléments nouveaux, ce qui se traduit par une augmentation de $50 \%$ des opportunités présentes dans le premier SWOT.

\section{Contrairement aux deux précédents ateliers, celui traitant du nouveau marché régional de la sous-traitance est demeuré très limité en termes d'apprentissage collectif.}

